\title{
Aberrant expression of the UPF1 RNA surveillance gene disturbs keratinocyte homeostasis by stabilizing $A R E G$
}

\author{
YAOJIA CHENG ${ }^{1 *}$, QIUPING LU ${ }^{1 *}$, NANNAN SHI ${ }^{1 *}$, QIONGYAN ZHOU ${ }^{2 *}$, \\ JINGJING RONG ${ }^{3}$, LIYUN LI ${ }^{4}$, LI WANG ${ }^{1}$ and CHEN LIU ${ }^{1}$ \\ ${ }^{1}$ Institute of Aging Research, School of Medicine, Hangzhou Normal University, Hangzhou, Zhejiang 311121; \\ ${ }^{2}$ Department of Dermatology, Affiliated Hospital, Ningbo University, Ningbo, Zhejiang 315211; \\ ${ }^{3}$ Institute of Zoology, Chinese Academy of Sciences, Beijing 100101; ${ }^{4}$ Information Centre, \\ First Affiliated Hospital of Kunming Medical University, Kunming, Yunnan 650032, P.R. China
}

Received June 14, 2019; Accepted January 22, 2020

DOI: $10.3892 /$ ijmm.2020.4487

\begin{abstract}
The up-frameshift suppressor 1 homolog (UPF1) RNA surveillance gene is a core element in the nonsense-mediated RNA decay (NMD) pathway, which impacts a broad spectrum of biological processes in a cell-specific manner. In the present study, the contribution of the NMD pathway to psoriasis lesions and its moderating effects on the biological processes of keratinocytes was reported. Sanger sequencing for skin scales from two patients with psoriasis identified two mRNA mutations (c.2935_2936insA and c.2030-2081del) in the UPF1 gene. The somatic mutants produced truncated UPF1 proteins and perturbed the NMD pathway in cells, leading to the upregulation of NMD substrates. As the most abundant epidermal growth factor receptor ligand in keratinocytes, it was concluded that amphiregulin (AREG) mRNA is a natural NMD substrate, that is dependent on its $3^{\prime}$ untranslated region sequence. Perturbed NMD modulated keratinocyte homeostasis in an $A R E G$-dependent but nonidentical manner, which highlighted the unique characteristics of NMD in keratinocytes. By targeting AREG mRNA post-transcriptionally, the UPF1-NMD pathway contributed to an imbalance between proliferation on the one hand, and apoptosis and abnormal differentiation, migration and inflammatory response on the other, in keratinocytes, which indicated a role of the NMD pathway in the full development of keratinocyte-related morbidity and skin diseases.
\end{abstract}

Correspondence to: Professor Li Wang or Dr Chen Liu, Institute of Aging Research, School of Medicine, Hangzhou Normal University, 2318 Yuhangtang Road, Hangzhou, Zhejiang 311121, P.R. China

E-mail: liwang@hznu.edu.cn

E-mail: chenl@hznu.edu.cn

${ }^{*}$ Contributed equally

Key words: UPF1, somatic mutation, keratinocyte, AREG, mRNA degradation, post-transcriptional

\section{Introduction}

Nonsense-mediated RNA decay (NMD) is a highly conserved eukaryotic mRNA surveillance mechanism that eliminates transcripts with a premature termination codon (PTC) located $>50-55 \mathrm{nts}$ upstream of the last exon-exon junction (EJ), resulting from mutations or aberrant splicing $(1,2)$. In addition to the targeting of PTC-harboring mRNAs for surveillance, $\sim 5-30 \%$ of cellular transcripts are finely degraded by the NMD pathway as natural substrates for modulating biological processes $(3,4)$; therefore, a disrupted NMD may be exploited to cause gene expression disruption without quality-control and fine-tuning (5). In addition, NMD impacts the cellular transcriptome in cell-type specific manners, indicating that NMD perturbation has a selective effect in increasing the levels of reported NMD substrates, such as NF- $\mathrm{k}$-B-inducing kinase (NIK), transducing $\beta$ like 2 (TBL2) and $\mathrm{N}$-acetyltransferase 9 (NAT9), in different cell lines $(6,7)$. The up-frameshift suppressor 1 homolog (UPF1) gene, encoding an ATP-dependent RNA helicase, is the central regulator of the NMD pathway (8). A feedback loop between the NMD and the UPF1 gene has been reported, in which UPF1 protein was rate-limiting for NMD and responses to NMD perturbation (6). UPF1 mutations have been deemed to play a causal role in malignant tumors, such as pancreatic adenosquamous carcinomas and inflammatory myofibroblastic tumors in our previous studies, suggesting that perturbed NMD is involved in cell hyperproliferation, abnormal differentiation and stable activation of inflammation $(7,9)$. The modulation of the NMD pathway has been suggested to provide a benefit in several disease therapies $(5,10)$, and therefore understanding the interactions between NMD and diseases can optimize preventive and therapeutic approaches against diseases. Herein, two somatic UPF1 mutations (c.2935_2936insA and c.2030-2081del) were identified in two patients with sporadic psoriasis scales, which perturbed the ability of the UPF1-NMD pathway to degrade substrates in keratinocyte cells. In the present study, to the best of our knowledge, mutated UPF1 transcripts in psoriasis have been discovered for the first time, which indicated a role of the NMD pathway in skin disease. 
A previous study hypothesized that there are keratinocyte-specific targets of the NMD pathway, and the amphiregulin (AREG) gene, which encodes the most abundant epidermal growth factor receptor (EGFR) ligand in keratinocytes, was suggested as one such substrate (11). AREG is overexpressed in a wide spectrum of epithelial diseases, including squamous cell carcinoma of the head and neck, colon cancer, lung cancer and psoriasis (12-15). AREG transgenic animals show epidermal acanthosis, keratinocyte hyperproliferation, hyperkeratosis, cutaneous immune cell infiltration and angiogenesis in the skin $(16,17)$. However, it is unknown how $A R E G$ is regulated post-transcriptionally by mRNA decay. In the present study, to the best of our knowledge, it is the first time that the potential mechanism of the AREG-NMD axis involved in keratinocyte cells was explored. In addition, cell activities such as differentiation, wound healing and inflammatory response, which are highly relevant to the pathogenesis of numerous skin diseases, were demonstrated to be regulated by UPFI in an $A R E G$-dependent manner. This finding has created an opportunity to better understand the NMD pathway in keratinocytes, and indicates role of the NMD pathway in the development of diseases associated with keratinocyte morbidities.

\section{Materials and methods}

Statistical analysis. The gene expression profiles of human paired psoriasis tissues were retrieved from Gene Expression Omnibus (GEO) database. After carefully screening the content, discarding the datasets with incomplete information and those lacking control patients, the 5 datasets with paired samples, GDS5392, GDS4602, GDS2518, GDS3539, and GDS4600, were obtained (18-22). R packages (version 3.5.2) were used to annotate the raw data and make the expression matrix (23). The median of expression level was chosen for genes matched by several probes, and paired $t$ test was used to compare UPF1 expression. Student's t-test was used for the analysis of statistical significance between two groups, and Bonferroni test was used for multiple comparisons after the analysis of variance. Data for continuous variables are expressed as the mean \pm SD. All statistical analyses were performed using SPSS 21.0 software (IBM Corp.). P $<0.05$ from a two-tailed test was considered to indicate a statistically significant difference.

Clinical samples. A total of 10 patients with a dermatologist-confirmed diagnosis of vulgaris psoriasis, according to the 'Consensus on diagnosis and treatment of Chinese integrative medicine for psoriasis vulgaris', published in China $(24,25)$, were recruited at the Affiliated Hospital of Ningbo University (Ningbo, China) between January 2016 and July 2017. The patient information is shown in Table SI. The lesion scales from 6 patients (P1-P6) were independent samples, while the lesion scales and adjacent healthy samples from 4 patients (PA-PD) were paired samples. All the psoriasis patients had no other autoimmune or systemic diseases, and required a typical lesion of $\geq 1 \mathrm{~cm}$ in size that was suitable for biopsy, and the target lesion and surrounding $5 \mathrm{~cm}$ area were not treated with any therapeutic measures for at least 2 weeks before the sampling. Skin scales or healthy cornified epidermal layer of patients with psoriasis vulgaris were scraped by blunt scalpels with patients' permission. The scales were promptly soaked in liquid-free nitrogen RNA sample storage solution (Beijing Bomaide Gene Technology Co., Ltd.).

Imiquimod (IMQ)-induced psoriasis-like mice. To create the well-established psoriasis-like skin model (26), 8-11-weeks-old $\mathrm{Balb} / \mathrm{c}$ mice provided by the Experimental Animal Center of Hangzhou Normal University were kept under standard laboratory conditions of 12-h light-dark cycles, $50 \%$ humidity and $24-26^{\circ} \mathrm{C}$ ambient temperature with free access to food and water. The mice ( 2 male and 2 female, $20-25 \mathrm{~g}$ ) received a daily topical dose of $40 \mathrm{mg}$ commercially available IMQ cream (5\%; Zhuhai United Laboratories Co., Ltd.) on the shaved back, equivalent of a daily dose of $2.083 \mathrm{mg}$ of the active compound. The control shaved skin nearby the IMQ-treated area (distance, $1.5-2 \mathrm{~cm}$ ) was treated similarly with a control cream (Vaseline Lanette cream; Fagron). During the 7-day experiment, the topical IMQ treatment did not lead pain, and the health of all 4 mice was monitored daily. On day 7, all 4 mice were sacrificed by rapid cervical vertebra dislocation, and the skin specimens of mice were collected once the cervical tissue separation was ensured.

Reverse transcription-quantitative PCR $(R T-q P C R)$ and $m R N A$ sequencing. Total RNA from model mice, clinical tissues or cells was isolated using TRIzol ${ }^{\circledR}$ reagent (Invitrogen; Thermo Fisher Scientific, Inc.), and first-strand cDNA for RT-qPCR was synthesized using the PrimeScript ${ }^{\mathrm{TM}} \mathrm{RT}$ reagent kit with gDNA Eraser according to the manufacturer's protocol (Takara Bio, Inc.). qPCR analysis was performed using the QuantStudio $^{\text {TM }} 7$ Flex Real-Time PCR system (Thermo Fisher Scientific, Inc.) using SYBR Premix Ex Taq (Takara Bio, Inc.) with gene-specific primers listed in Table SII. The following thermocycling conditions were used: Initial denaturation at $95^{\circ} \mathrm{C}$ for $1 \mathrm{~min}$; followed by 40 cycles of $95^{\circ} \mathrm{C}$ for $15 \mathrm{sec}$ and $60^{\circ} \mathrm{C}$ for $30 \mathrm{sec}$. The disassociation stage was added to check the amplicon specificity. For transcript detections in scales tissues, $U P F 1$ was quantified using the allele-specific primers UPF1 insA for c.2935_2936insA mutant, or UPF1 del corresponding to the c.2030-2081del UPF1 mRNA (Table SII). Specificity and efficiency of allele-specific PCR were tested with corresponding UPF1 constructs, and further details are described in Data S1. Each mRNA quantification value represented an average of at least three measurements, and mRNA expression levels were calculated using the $2^{-\Delta \Delta C q}$ method (27) after normalization to the endogenous housekeeping gene $18 \mathrm{~S}$ for human samples or GAPDH for mouse samples.

For RT-PCR, RT was performed using $1 \mu \mathrm{g}$ of total RNA with the PrimeScript ${ }^{\mathrm{TM}}$ 1st Strand cDNA Synthesis kit according to the manufacturer's protocol (Takara Bio, Inc.). PCR amplification was performed with nested primers (Table SIII) overlapping the coding sequence region of the UPF1 mRNA (NCBI Reference Sequence, NM_002911.3). The following thermocycling conditions were used: Initial denaturation at $96^{\circ} \mathrm{C}$ for $2 \mathrm{~min}$; followed by 35 cycles of $96^{\circ} \mathrm{C}$ for $10 \mathrm{sec}, 55^{\circ} \mathrm{C}$ for $30 \mathrm{sec}$ and $72^{\circ} \mathrm{C}$ for $2 \mathrm{~min}$. The sanger sequencing was performed at Tsingke Biological Technology.

Protein detection. For immunohistochemical (IHC), skin tissue from the model mice was fixed in $10 \%$ formalin for $24 \mathrm{~h}$ 
at room temperature and embedded in IHC-grade paraffin. Formalin-fixed paraffin-embedded sections were cut into 4-6- $\mu \mathrm{m}$ sections with a microtome and deparaffinized two times in xylene, followed by serial dilutions of ethanol. After heat-induced antigen retrieval using the antigen unmasking solution (Vector Laboratories, Inc.; Maravai LifeSciences) for $30 \mathrm{~min}$ at $95^{\circ} \mathrm{C}$, the internal peroxidase activity was quenched by incubation with $3 \%$ hydroperoxide in methanol for $15 \mathrm{~min}$ at room temperature, then sections were incubated in $3 \%$ bovine serum albumin (Beyotime Institute of Biotechnology) for $1 \mathrm{~h}$ at room temperature. The prepared sections were incubated overnight at $4^{\circ} \mathrm{C}$ with anti-UPF1 primary antibodies (cat. no. sc-390096; 1:100; Santa Cruz Biotechnology, Inc.). The secondary antibody (cat. no. GAMPO; 1:1; DAKO; Agilent Technologies, Inc.) was used according to the manufacturer's instructions for $50 \mathrm{~min}$ at room temperature. The intensity of IHC staining was measured using ImageJ software (version 1.52a; Media Cybernetics, Inc.).

Protein extraction for western blotting was performed using RIPA lysis buffer (Beyotime Institute of Biotechnology) with phenylmethylsulfonyl fluoride serine protease inhibitor (Beyotime Institute of Biotechnology). Protein extracts were quantified using a BCA Protein Assay kit (Beyotime Institute of Biotechnology) according to the manufacturer's instructions. Total proteins $(30 \mu \mathrm{g})$ were resolved by SDS-PAGE ( $8 \% \mathrm{gel})$ and visualized using Odyssey ${ }^{\circledR}$ imaging system with IRDye secondary antibodies (cat. no. 926-32210; 1:1,000; LI-COR Biosciences) for $15 \mathrm{~min}$ at room temperature. The following primary antibodies were used overnight at $4^{\circ} \mathrm{C}$ : Anti-UPF1 (cat. no. sc-166092; 1:200; Santa Cruz Biotechnology, Inc.), anti-AREG (cat. no. GTX100986; 1:200; GeneTex, Inc.), and anti-GAPDH (cat. no. M20006L; 1:1,000; Abmart Pharmaceutical Technology Co., Ltd.). Semi-quantification was performed using ImageJ software.

Cell culture and transfection. Primary cultured normal human keratinocytes $(\mathrm{HEK} \alpha)$, immortalized nontumorigenic human keratinocyte-derived cell line ( $\mathrm{HaCaT})$ and 293T cell line were purchased from the Shanghai Institute of Biochemistry and Cell Biology. HaCaT and 293T cells were cultured in DMEM with 10\% FBS (Biological Industries), and HEK $\alpha$ cells were cultured in EpiLife Medium with $60 \mu \mathrm{M}$ calcium with Keratinocyte Growth Supplement (Gibco; Thermo Fisher Scientific, Inc.). All cells were incubated at $37^{\circ} \mathrm{C}$ in a humidified $5 \% \mathrm{CO}_{2}$ incubator.

For plasmid and small interfering (si)RNA transfection, $4 \times 10^{4}$ cells/well in 6-well plates were cultured overnight and then transfected using the GeneTran Reagent (Biomiga, Inc.). For co-transfection targeting both UPFI and AREG, constructs were transfected at the same time; however, when constructs targeting the same gene were co-transfected, construct for knockdown was transfected first, then the overexpression construct was transfected $24 \mathrm{~h}$ later. Total mRNA and protein of cells were collected $48 \mathrm{~h}$ after transfection.

Constructs and oligonucleotides. A short hairpin RNA (shRNA) sequence specifically targeting UPF1 (Table SIV) was cloned into Phblv-U6-puro (provided by Professor Fan Handong, Hangzhou Normal University) by BamHI and EcoRI digestion. The siRNA targeting AREG (targeted sequence,
CCACAAATACCTGGCTATA) and a scrambled sequence were purchased from Guangzhou RiboBio Co., Ltd. The UPF1 insA and UPF1 del plasmids were constructed based on the pCMV-MYC-UPF1 vector (provided by Professor Lynne E. Maquat, University of Rochester). Primers for site-directed mutagenesis (Table SIV) were designed by QuickChange Primer Design (Agilent Technologies, Inc.) and applied with the KOD-Plus-PCR enzyme (Toyobo Life Science). Residual templates were digested by DpnI (New England Biolabs, Inc.) at $37^{\circ} \mathrm{C}$ for $5 \mathrm{~h}$. The AREG gene open reading frame (ORF) with or without the $3^{\prime}$ untranslated region (3'UTR), which was referred as $A R E G-3$ 'UTR-pEGFP or AREG-ORF-pEGFP, respectively, was cloned into the pEGFP-N1 vector (provided by Dr Wang Miao, Hangzhou Normal University) using the Hind III and BamHI restriction sites. The primer sequences used are listed in Table SIV. The AREG 3'UTR sequence was amplified and cloned into the dual-luciferase reporter construct pEZX-FR02 (GeneCopoeia, Inc.) by double digestion with EcoRI and SpeI (the primer sequences used are listed in Table SIV) to generate the pEZX-AREG-3'UTR construct.

Half-life analysis. The 293T cells were transfected with or without shUPFl and the transcription in cells was inhibited by incubation with $20 \mathrm{mg} / \mathrm{ml} 5,6$-dichloro-1- $\beta$-Dribofuranosylbenzimidazole (DRB; Enzo Life Sciences, Inc.) dissolved in DMSO. Total RNA was extracted at 0, 3, 5, 7, and $10 \mathrm{~h}$ following the inhibition of transcription, and AREG mRNA levels were measured by RT-qPCR, as described above. Quantity of RNA at each time point was determined by comparison with standard curves generated by amplification of the time-zero RNA sample.

Dual-luciferase reporter assays. The pEZX-AREG-3'UTR transfected $293 \mathrm{~T}$ cells were co-transfected with or without shUPF1 using the GeneTran ${ }^{\mathrm{TM}}$ III reagent (Biomiga, Inc.). At $80 \%$ confluence and $48 \mathrm{~h}$ post transfection, cells were collected and the activities of both firefly luciferase (FLuc) and Renilla luciferase (RLuc) were measured according to the instructions of the dual luciferase reporter assay system (GeneCopoeia, Inc.). The internal standard for transfection efficiency was normalized to RLuc activity.

Cell proliferation and migration assays. HaCaT and HEKa cells were plated at a density of $2 \times 10^{4}$ cells/well in 96-well plates and incubated overnight. The proliferation index was measured using a Cell Counting Kit-8 (Dojindo Molecular Technologies, Inc.) at $48 \mathrm{~h}$ according to the manufacturer's instructions. For the cell wound healing assay, cells in the logarithmic growth phase were seeded in 24 well plates at a density of $5 \times 10^{5}$ cells/ well. When the cells reached $100 \%$ confluence, a straight line was drawn in the cells in each well with a $10-\mu 1$ pipette tip and the cells were serum-starved. Representative images (magnification, x20) of wound closure were captured at 0,6,12, 24 , and $48 \mathrm{~h}$ with an inverted microscope, and the wound area was measured using Image J software. The percentage of wound coverage was calculated by the difference in area between indicated time point and $0 \mathrm{~h}$, divided by the area at $0 \mathrm{~h}$.

Accession codes. The gene sequences used in the current study are publicly available at the GenBank [UPF1 mRNA 

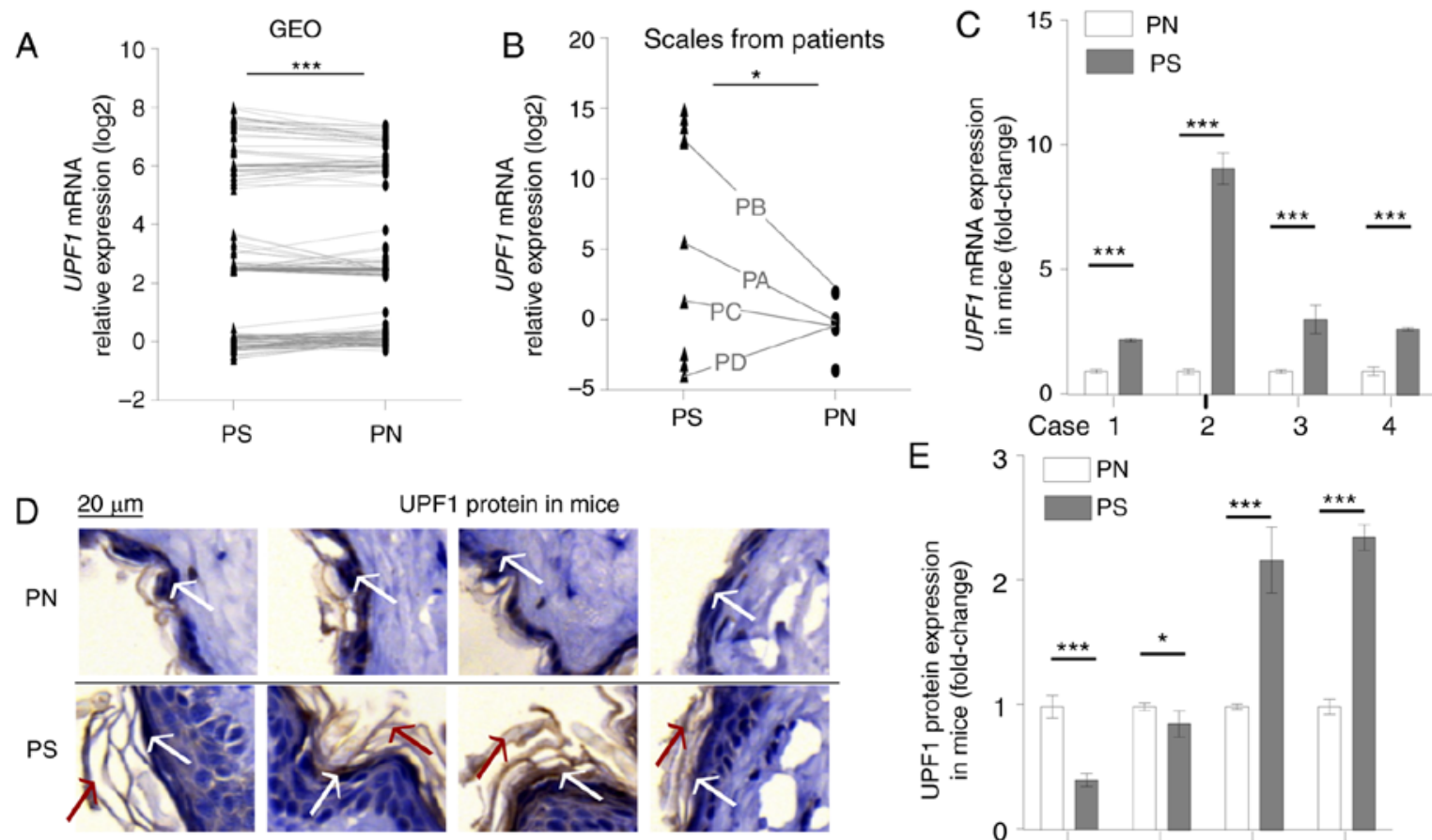

UPF1 protein in mice

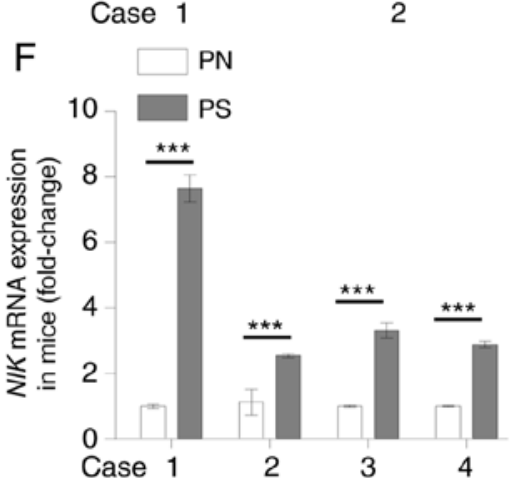

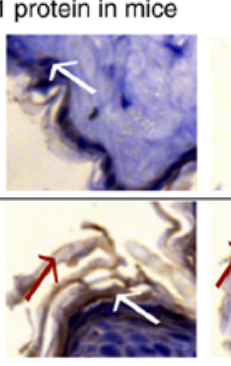

3

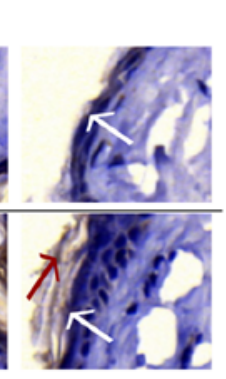

4
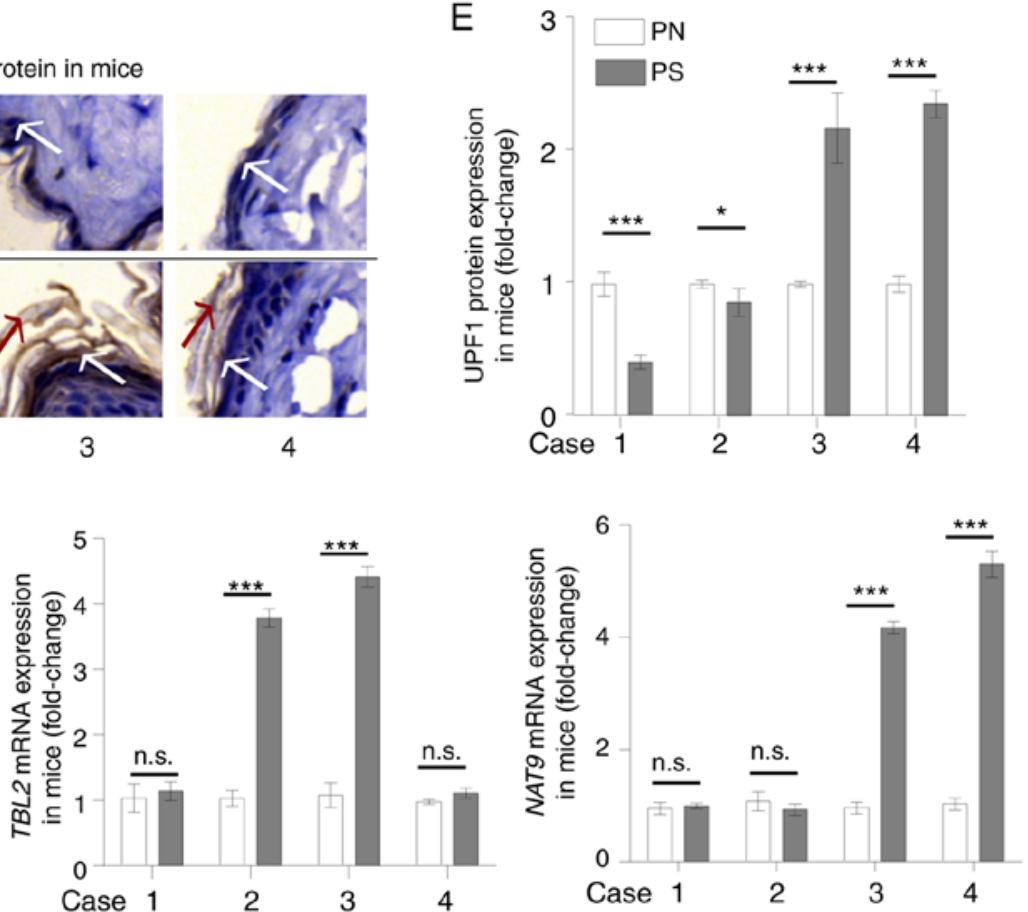

Figure 1. Abnormal expression of UPF1 in psoriasis. (A) Paired sample t-test for 186 paired psoriasis samples from GEO datasets (GDS5392, GDS4602, GDS2518, GDS3539 and GDS4600). (B) Student's t-test for UPF1 mRNA levels in 10 psoriasis scales and 8 healthy cornified epidermal layer samples. Among the samples, four paired tissues were indicated with black lines. The UPF1 expression increased in the psoriasis group; 3/4 paired samples showed an upregulated expression level of UPF1. (C) Mice received a daily topical IMQ cream to induce psoriasis-like skin. The expression of UPF1 increased in all four psoriasis-like tissues, as compared with the paired normal skin tissue samples. (D) The expression of UPF1 protein distributed throughout the cytoplasm in mouse skin was detected by IHC. The white arrows indicate positive UPF1 protein staining, and the red arrows indicate skin hyperkeratosis in IMQ-treated samples. (E) IHC staining was suantified using ImageJ software. The UPF1 protein decreased in $2 / 4$ paired samples. (F) The mRNA expression of three NMD substrates, NIK, TBL2 and NAT9, were higher in psoriasis-like skin compared with normal tissue. Data are presented as the mean \pm SD from three independent experiments. ${ }^{*} \mathrm{P}<0.05$ and ${ }^{* * * *} \mathrm{P}<0.001$. n.s., not significant; $U P F 1$, up-frameshift suppressor 1 homolog; IMQ, imiquimod; IHC, immunohistochemistry; NMD, nonsense-mediated RNA decay; NIK, NF-к-B-inducing kinase; TBL2, transducing $\beta$ like 2; NAT9, N-Acetyltransferase 9; PS, psoriasis or IMQ-treated psoriasis-like skin; PN, normal cornified epidermal layer.

(NM_002911.3) and AREG mRNA (NM_001657.4)]. Data on UPF1 mutations were deposited in the GenBank under accession numbers MH183202 (UPF1 c.2935_2936 insA) and MK089816 (UPF1 c.2030_2081 del 50 nts).

\section{Results}

Abnormal expression of UPF1 in psoriasis and psoriasis-like mouse model. First, a paired samples test for UPF1 expression was concluded using GEO datasets GDS5392, GDS4602, GDS2518, GDS3539 and GDS4600. An analysis of 186 paired psoriasis and corresponding normal tissues showed that the UPF1 mRNA expression increased significantly in psoriasis (Fig. 1A). Increased UPF1 mRNA levels were also observed in sporadic patients' skin scales obtained in the current study, including four paired tissues (PA, PB, PC and PD; Fig. 1B), six independent scales and four independent normal tissues. The IMQ induced psoriasis-like skin showed morphological characteristics consistent with the symptoms of human psoriasis (28), such as skin thickening (Fig. S1 and Data S1) and hyperkeratosis (Fig. 1D), and RT-qPCR showed that the psoriasis-like skin expressed higher mRNA levels of proinflammatory cytokines and chemokines associated with psoriasis including $A R E G$, interleukin $(I L)-17 A, I L-6$, tumor necrosis factor- $\alpha$ and $C-X-C$ motif chemokine 2 (data not shown). All 4 paired tissues from IMQ-induced psoriasis-like skin exhibited increased UPF1 mRNA levels (Fig. 1C), while protein levels were significantly decreased in $2 / 4$ pairs, as detected by IHC (Fig. 1D and E). Abnormal UPF1 expression levels were also revealed by GEO database analysis for three other skin 

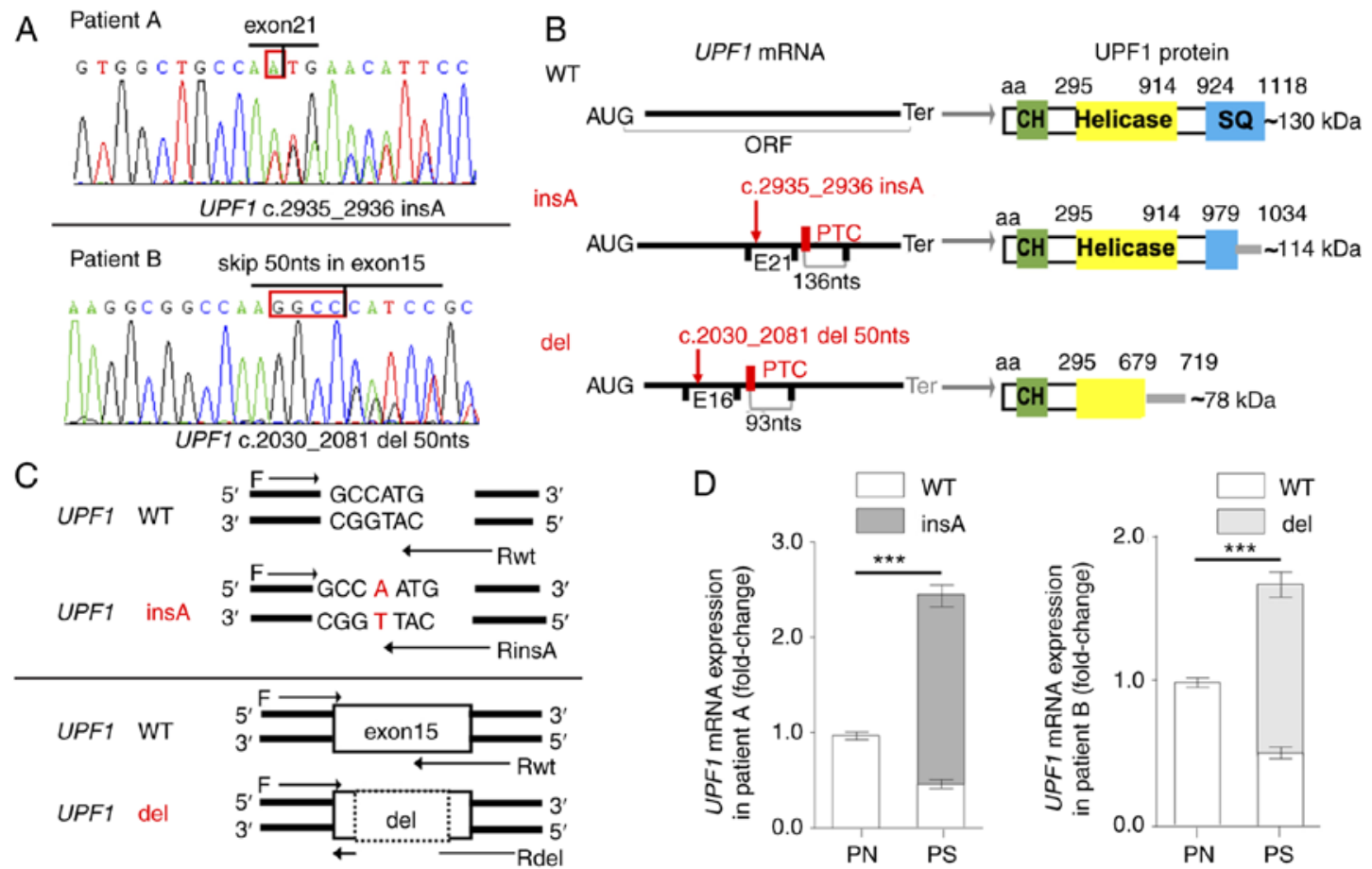

Figure 2. Somatic mutations of UPF1 transcripts in patients with psoriasis vulgaris. (A) Sequencing traces corresponding to UPF1 insA (GenBank ID, MH183202) in patient A and UPF1 del (GenBank ID, MK089816) in patient B. The red frame indicates the insertion or shared sequence for splicing. The sequencing primers used were F4/R4 and F3/R3 from Table SIII. (B) Schematic representation of the aberrant mRNAs and proteins. The mutations and PTCs are indicated in red, while the EJs and Ters are presented in black in the mRNA schematic. The functional domains of UPF1 are shown in the protein schematic, and the gray lines indicate that the amino acid sequence was changed until the Ter. (C) Primers specific to UPF1 mutants or UPF1 WT were designed to detect the expression of UPF1 transcripts in the 2 patients. (D) The portions of UPF1 insA and UPF1 del were evaluated by reverse transcription-quantitative PCR. Mutated $U P F 1$ transcripts were only detectable in the psoriasis scales. Data are presented as the mean $\pm \mathrm{SD}$ from three independent experiments. ${ }^{* * *} \mathrm{P}<0.001$. UPF1, up-frameshift suppressor 1 homolog; PTC, premature termination codon; EJ, exon-exon junction; Ter, termination codon; WT, wild-type; PS, psoriasis scales; PN, normal cornified epidermal layer; SQ, serine-glutamine; $\mathrm{CH}$, calponin homology.

diseases tissues, including the Marfan syndrome, squamous cell carcinoma and melanoma tissues (Fig. S2 and Data S1). As UPF1 mRNA is a natural substrate of the NMD pathway, as well as a rate limiting element for NMD (6), the abnormal expression of UPF1 in those skin disease tissues was presumed to indicate a disturbed NMD pathway in the keratinocyte cells. According to the cell-type specific manner of NMD, multiple reported NMD substrates, which have been reported to be sensitive to $U P F 1$ depletion in different cell lines (6-8), were tested by RT-qPCR in shUPF1 treated keratinocyte cells in a preliminary study. Three well-characterized endogenous NMD substrates, NIK, TBL2 and NAT9 mRNAs, were verified to increase in level in response to UPF1-depletion in the studied 293T and keratinocyte cells (data not shown), thus, the NIK, TBL2 and NAT9 mRNAs were selected to presented the NMD endogenous substrates. In the psoriasis-like mouse model, a disturbed NMD was hinted by the accumulation of NIK, TBL2 and NAT9 mRNAs in some paired lesions (Fig. 1F).

Aberrant transcripts of UPF1 in psoriasis scales. The skin scales are mostly composed of corneocytes (29); thus, the tissues may have been fragile for RNA sequencing. Five out of ten psoriasis scales from sporadic psoriasis patients were demonstrated to be reliable sources of RNA, as PCR fragments of UPF1 and AREG mRNAs were amplified successfully in these tissues (Fig. S3 and Data S1). Among the 5 available scales for RNA sequencing, 2 aberrant UPF1 transcripts were identified in patients $A$ and $B$, and neither mutant was detected in the corresponding healthy epidermal tissue. The heterozygous mutation c.2935_2936insA (Fig. 2A) identified in patient A (a 36-year-old man with an affected buttock) generated an in-frame PTC located 136 nts upstream of the exon 22-23 junction (Fig. 2B). The heterozygous mutation c.2030-2081del (Fig. 2A) in patient B (a 48-year-old woman with affected arms) was spliced by the use of noncanonical splice donor/acceptor within exon 15 sharing a GGCC sequence, which generated a PTC 93 nts upstream of the exon 16-17 junction (Fig. 2B). There was no detectable mutation in the corresponding DNA or flanking introns, and, to the best of our knowledge, these two mutations had not been reported prior to the present study. The canonical ORF of UPF1 was disrupted in both aberrant transcripts, and the mutants were predicted to produce truncated proteins losing serine-glutamine cluster domain or the helicase domain (Fig. 2B).

To analyze the expression of UPF1 transcripts in psoriasis scales, specific primers were designed, according to the aberrant sequences (Fig. 2C). The specificity and efficiency of RT-qPCR for UPF1 ins A were tested with allele-specific amplification. Transcript analysis demonstrated that the aberrant mutants were only detectable in psoriasis scales, whereas the wild-type (WT) transcripts were expressed in both the scale lesions and healthy cornified epidermal layer, showing a decrease in lesions (Fig. 2D).

Perturbation of NMD results from UPF1 insA and UPF1 del transcripts. The selective accumulation of the PTC-harboring transcripts in lesions but not in the adjacent normal tissues 
A

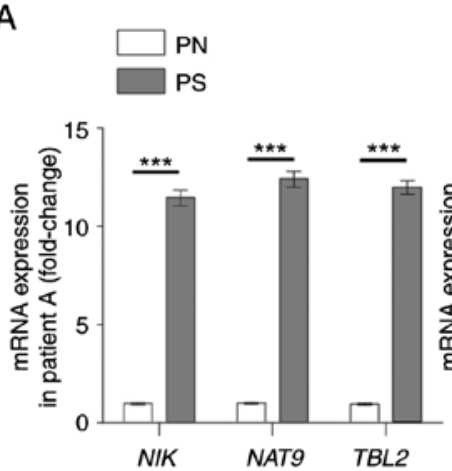

C
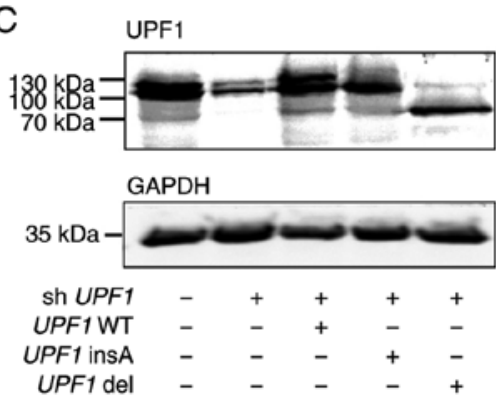

B

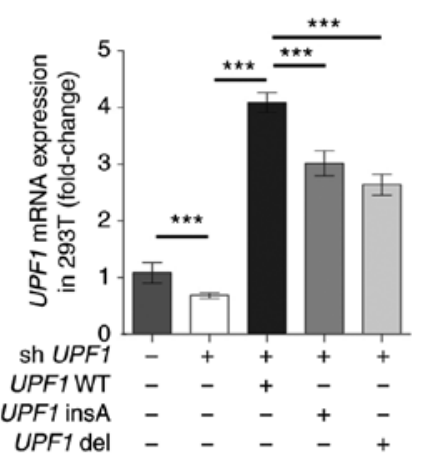

D
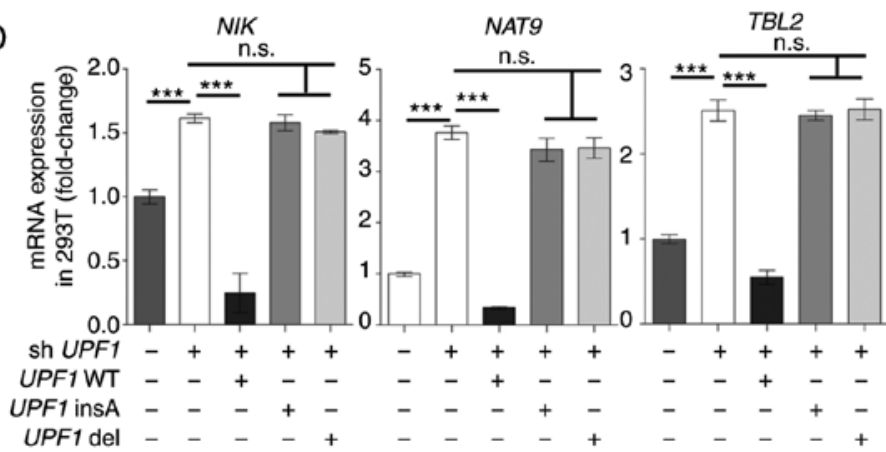

Figure 3. Aberrant UPF1 transcripts disrupt the function of the NMD pathway. (A) The expression level of NIK, TBL2 and NAT9 showed an increase in psoriasis scales from patients A and B compared with PN samples. (B) 293T cells were transfected with the shUPF1 construct, and with the UPF1 WT, $U P F 1$ insA or $U P F 1$ del vector $24 \mathrm{~h}$ later. $U P F 1$ transcript expression was quantified $48 \mathrm{~h}$ after transfection. The expression levels of $U P F 1$ insA and $U P F 1$ del were lower than that of UPF1 WT following transfection with the respective constructs. (C) Western blot analysis was used to measure the expression of UPF1 protein isoforms, and GAPDH was used as a control. The UPF1 insA and UPF1 del generated minor protein bands, the predicted molecular weights are indicated. (D) mRNA expression levels of NMD substrates NIK, TBL2 and NAT9 were increased in UPF1-depleted 293T cells, as detected by reverse transcription-quantitative PCR. Data are presented as the mean \pm SD from three independent experiments. ${ }^{* * *} \mathrm{P}<0.001$. n.s., not significant. $U P F 1$, up-frameshift suppressor 1 homolog; NMD, nonsense-mediated RNA decay; NIK, NF-к-B-inducing kinase; TBL2, transducing $\beta$ like 2; NAT9, N-Acetyltransferase 9; WT, wild-type; PS, psoriasis scales; PN, normal cornified epidermal layer.

raised the possibility that the NMD pathway was perturbed in the two scales. Consistent with this hypothesis, it was found that the expression level of preselected NMD substrates, NIK, $T B L 2$ and NAT9, was elevated in the psoriasis scales compared with normal control samples (Fig. 3A).

Successful overexpression and knockdown of UPF1 was confirmed (Fig. S5A and Data S1). The subsequent transfection of the UPF1 insA, UPF1 del or UPF1 WT constructs into UPF1-depleted $293 \mathrm{~T}$ cells revealed that the mutated transcripts were expressed at a slightly lower steady-state level than UPF1 WT at both the mRNA and protein levels (Fig. 3B and C; lanes 3-5). As predicted above, the mutants generated minor protein bands that were predicted to be $\sim 114 \mathrm{kDa}$ for UPF1 insA and $78 \mathrm{kDa}$ for UPF1 del (Fig. 3C; lanes 4-5), as compared with the $128 \mathrm{kDa}$ full-length UPF1 protein (Fig. 3C; lane 3). The truncated UPF1 protein in psoriasis scales was not confirmed, as no protein sample could be collected. Subsequently, the expression levels of NMD substrates, which depended on mutated UPF1, were examined. Unlike UPF1 WT, which could rescue NMD activity in $U P F 1$-depleted cells to decrease the level of NMD substrates, $U P F 1$ insA and UPF1 del did not impact the NIK, TBL2 or NAT9 mRNA expression levels, indicating that the two mutants lacked a detectable NMD function (Fig. 3D).

Identification of AREG MRNA as an NMD substrate depend on the 3'UTR of AREG. After verifying the disruption of the
NMD pathway by UPF1 mutants, gene regulation under a deficient NMD pathway was explored. As NMD targets diverse substrates and governs RNA homeostasis in a cell-specific manner (6), it was hypothesized that NMD deficiency would allow partial targets to be stabilized, leading to a disordered gene expression in a keratinocyte-specific manner. As the most abundant EGFR ligand gene in keratinocytes (30), AREG mRNA was identified as a candidate for the NMD pathway, since it harbors an EJ in its 3'UTR, which could trigger moderate NMD (Fig. 4A) (1). The expression level of AREG mRNA was increased in psoriasis tissues and UPF1-depleted keratinocyte cells, while $U P F 1$ overexpression conversely decreased AREG mRNA levels in keratinocytes (Fig. 4B and C). Consistent with the well-established NMD substrates noted above, the dysregulation of $A R E G$ mRNA under UPF1 depletion could be rescued by UPF1 WT, but not by UPF1 insA or UPF1 del (Fig. 4D). This effect was also observed at the protein level, as shown by western blotting (Fig. 4E).

Given that transcript destabilization is the hallmark of direct NMD targets (31), RNA half-life analysis was performed on DRB-treated 293T cells with an inhibited transcriptional activity (32); it was found that AREG mRNA was stabilized $\sim 1.23$-fold in response to $U P F 1$ depletion (Fig. 5A). To verify the NMD-triggering effect of $A R E G$ mRNA, AREG vector with or without its 3 'UTR sequence was constructed (Fig. 5B), and the overexpression and knockdown of AREG was confirmed (Fig. S5B and Data S1). After knocking down 
A
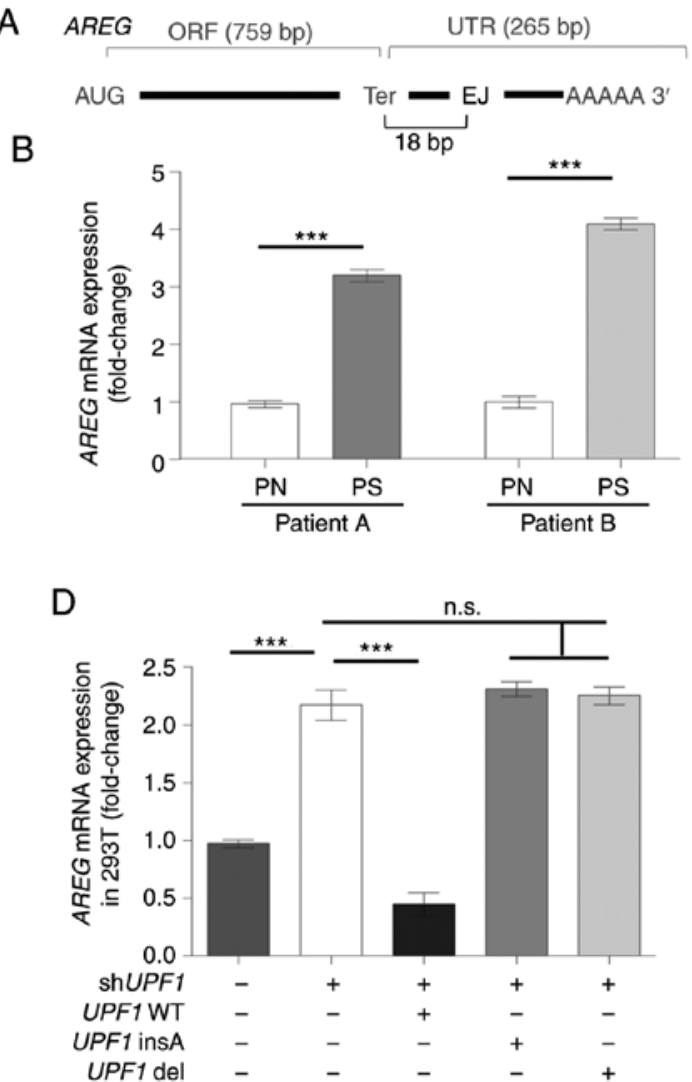

C
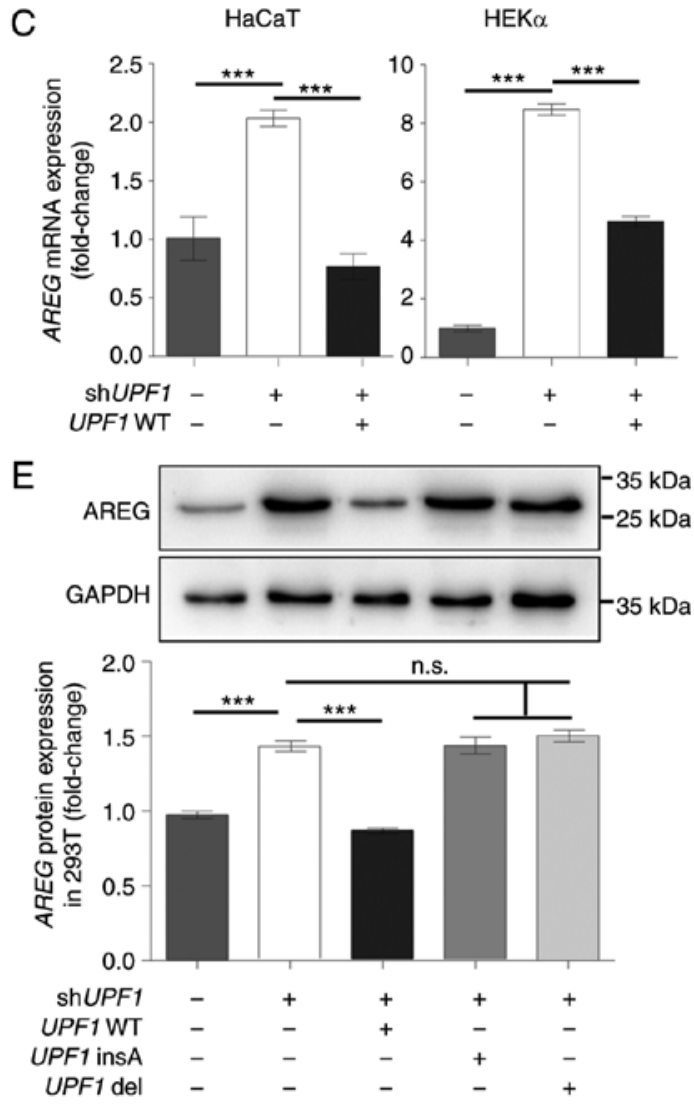

Figure 4. Expression of $A R E G$ is regulated by the NMD pathway. (A) Schematic representation of $A R E G$ mRNA. The EJ in the 3 'UTR was presumed to be an NMD pathway triggering feature. (B) $A R E G$ expression was increased in the psoriasis scales from patients A and B compared with their respective PN samples. (C) Keratinocytes were transfected with shUPF1 or UPF1 WT constructs. The AREG mRNA level was regulated by UPF1 expression in HEK $\alpha$ and $\mathrm{HaCaT}$ cells, as quantified $48 \mathrm{~h}$ after transfection. (D) $293 \mathrm{~T}$ cells were transfected with UPF1 expression vectors after transfection with shUPF1, and $A R E G$ mRNA levels were quantified $48 \mathrm{~h}$ after transfection. (E) AREG protein expression levels were measured by western blotting, and GAPDH was used as a control. AREG levels were only decreased by $U P F 1 \mathrm{WT}$. Data are presented as the mean $\pm \mathrm{SD}$ from three independent experiments. ${ }^{* * *} \mathrm{P}<0.001$. $A R E G$, amphiregulin; NMD, nonsense-mediated RNA decay; EJ, exon-exon junction; WT, wild-type; UPF1, up-frameshift suppressor 1 homolog; n.s., not significant; PS, psoriasis scales; PN, normal cornified epidermal layer.

endogenous UPF1 and AREG in 293T cells, no significant change was identified in the expression of exogenous $A R E G$ following transfection with AREG without the 3'UTR (Fig. 5C; lanes 5 and 6); however, the expression of $A R E G$ following transfection with AREG with 3'UTR was increased significantly (Fig. 5C; lanes 7 and 8), which indicated that the 3'UTR region was indispensable for NMD targeting. The current study further examined whether the 3'UTR of AREG was targeted by NMD via the dual-luciferase reporter system pEZX-FR02 (Fig. 5D). The results showed that the insertion of the AREG 3'UTR to pEZX-FR02 increased the FLuc activity following UPF1 depletion, indicating that this sequence was adequate for the triggering NMD (Fig. 5E).

$N M D$ regulates keratinocyte homeostasis by regulating AREG. UPF1 knockdown has been reported to result in cell apoptosis $(33,34)$, while $A R E G$ positively regulates the proliferation of keratinocytes (11). Successful target gene expression regulation by $U P F 1$ and $A R E G$ vectors was confirmed in keratinocyte cells (Fig. S6 and Data S1), and the cell morphology and area of cells did not change significantly (Fig. S7 and Data S1). The viability of UPF1-depleted HaCaT and HEK $\alpha$ cells was decreased, and the inhibition of AREG further decreased cell viability in these cells (Fig. 6A). These results were confirmed by determining the mRNA expression levels of proliferation marker protein Ki67, and BCL2 associated agonist of cell death $(B A D)$ (Fig. 6B). Epidermal acanthosis, hyperkeratosis and angiogenesis have been previously detected in the skin of AREG transgenic animals (35), which indicates an ability of $A R E G$ to affect cell differentiation. UPF1 depletion markedly inhibited the expression of the differentiation gene keratin-5 (K5), the terminal differentiation marker filaggrin (FLG) and keratin-10 (K10). The expression levels of the differentiation markers were reversed in HaCaT and HEK $\alpha$ cells when $A R E G$ inhibition was coupled with UPF1 depletion (Fig. 6C).

Keratinocytes in which terminal differentiation is halted undergo changes in cell migration, and keratinocytes can repair wounds partly by migration (36). A wound healing assay was therefore performed to estimate the extent to which UPF1 depletion promoted cutaneous wound healing. UPF1 depletion increased the ability of both $\mathrm{HaCaT}$ and $\mathrm{HEK} \alpha$ cells to migrate and re-epithelialize as demonstrated by the changes in cell-free areas. Following double knockdown of $A R E G$ and UPF1 in keratinocytes, the cell re-epithelialization was impaired compared with the shUPF1 group (Fig. 6D and E). The perturbed NMD in the shUPF1 group induced the expression of motility markers, cyclooxygenase-2 (COX-2), matrix 
A

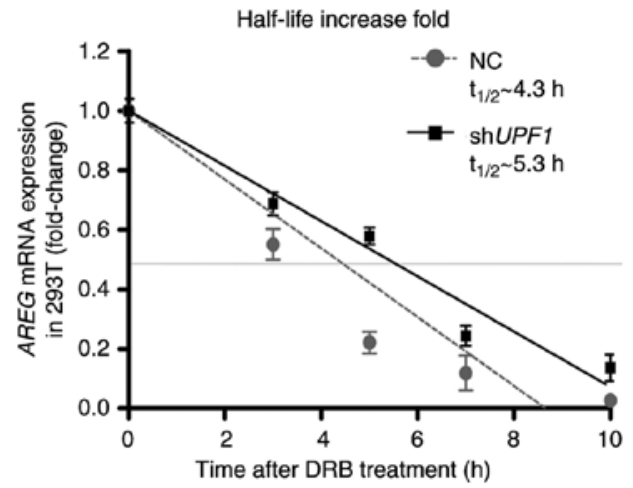

B

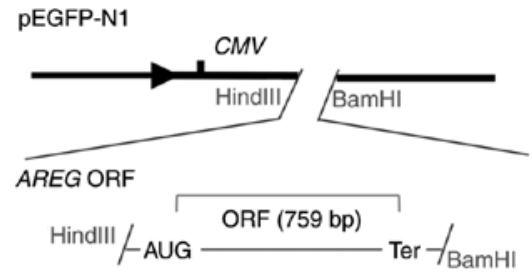

AREG-3'UTR

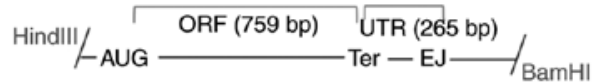

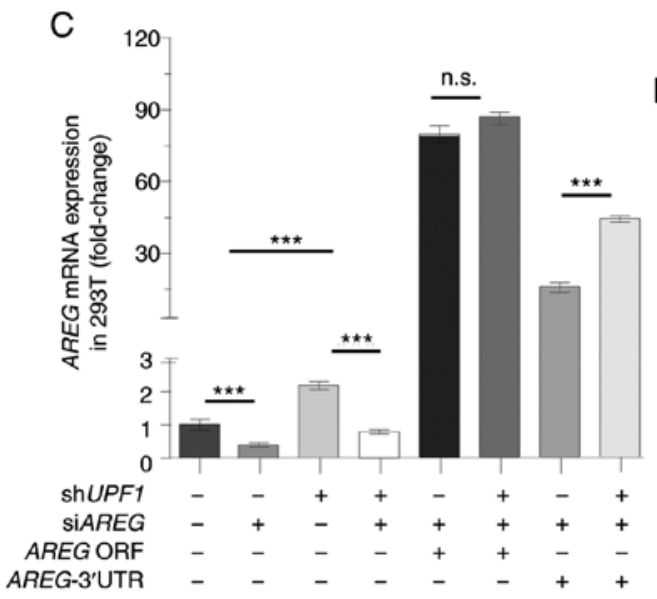

D

pEZX-FR02

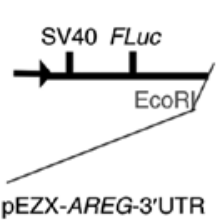

$$
\text { EcoRI/ Ter }
$$

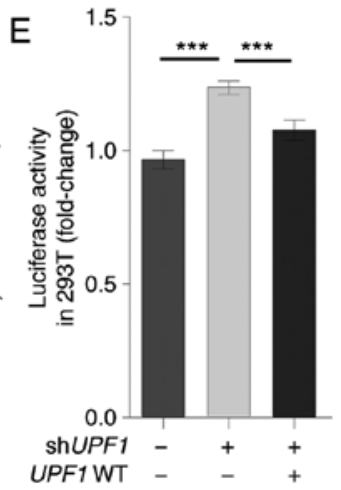

Figure 5. AREG triggers the NMD pathway post-transcriptionally, depending on its 3'UTR. (A) 293T cells were treated with DRB, and AREG mRNA levels were evaluated by RT-qPCR at $0,3,5,7$ and $10 \mathrm{~h}$. The $\mathrm{t}_{1 / 2}$ represents the half-life of $A R E G$ mRNA. The AREG mRNA was stabilized by UPF1 depletion, as detected by RT-qPCR. (B) The structures represent the expression vector for AREG ORF and AREG-3'UTR based on pEGFP-N1. (C) 293T cells were transfected with shUPF1, and AREG expression vectors were transfected $24 \mathrm{~h}$ later. AREG mRNA levels were quantified $48 \mathrm{~h}$ after transfection. The 3'UTR region was required for NMD triggering by AREG. (D) Schematic representation of the dual-luciferase reporter system pEZX-AREG-3'UTR. (E) 293T cells co-transfected with pEZX-AREG-3'UTR plasmid together with shUPF1 or UPF1 WT vector. Cells were collected and the FLuc activity was measured $48 \mathrm{~h}$ after transfection. RLuc activity was used as a control. The $3^{\prime}$ UTR region was sufficient to trigger the NMD pathway. Data are presented as the mean \pm SD from three independent experiments. ${ }^{* * *} \mathrm{P}<0.001$. AREG, amphiregulin; NMD, nonsense-mediated RNA decay; DRB, 5,6-dichloro-1- $\beta$-D-ribofuranosylben zimidazole; UPF1, up-frameshift suppressor 1 homolog; firefly luciferase, FLuc; Renilla luciferase, RLuc; ns, not significant; NC, normal cells; RT-qPCR, reverse transcription-quantitative PCR.

metalloproteinase $1(M M P 1)$ and snail family transcriptional repressor 1 (SNAII) compared with control group, while double knockdown of AREG and UPF1 lost this capacity (Fig. 6F). Keratinocytes also produce proinflammatory mediators that react against damage (37). In the current study, an increased expression of chemokine (C-C motif) ligand 20 (CCL2O) and human recombinant GRO- $\alpha$ (CXCL1) was observed following NMD disruption in HaCaT and HEK $\alpha$ cells. The increased expression of these proinflammatory chemokines following NMD disruption was restored by AREG knockdown in these cells (Fig. 6F).

\section{Discussion}

To investigate the NMD pathway in skin diseases, the core element $U P F 1$ was examined in patients with psoriasis and psoriasis-like mouse models. Psoriasis datasets from the GEO, human psoriasis scales and IMQ-induced skin inflammation samples all displayed increased UPF1 mRNA levels compared with their respective controls. In addition, analysis for several other skin diseases was conducted by analyzing UPF1 expression profiles acquired from the GEO database. An independent sample test for Marfan syndrome (GDS2960) showed a significantly increased $U P F 1$ expression in lesions compared with normal tissues, and multiple comparisons for squamous cell carcinoma (GDS2200) and melanoma (GDS1375) showed a high UPF1 expression in squamous cell carcinoma compared with actinic keratosis or normal tissues, and melanoma and benign nevi compared with normal tissues (26-38). A negative feedback regulatory network that directly acts on UPF1 in response to NMD perturbation has been identified in a previous study which demonstrated that $U P F 1$ itself is a natural substrate of the NMD pathway (6). Overexpression of UPF1 indicated by the GEO datasets analysis herein hinted at an abnormal NMD in several skin diseases besides psoriasis. Furthermore, although increased UPFI mRNA levels were observed in IMQ-induced psoriasis-like skin, this result was not confirmed at the protein levels in two mice. Expression levels of well-characterized NMD substrates, NIK, TBL2 and NAT9 mRNAs were also increased in mouse models of psoriasis, suggesting the possibility of UPF1-related NMD perturbation in psoriasis. The protein level of the NMD substrates was not determined in the current study, as the NMD pathway regulates substrates mainly by destabilizing the mRNAs, while protein level may be affected by translation efficiency or protein degradation (32). 

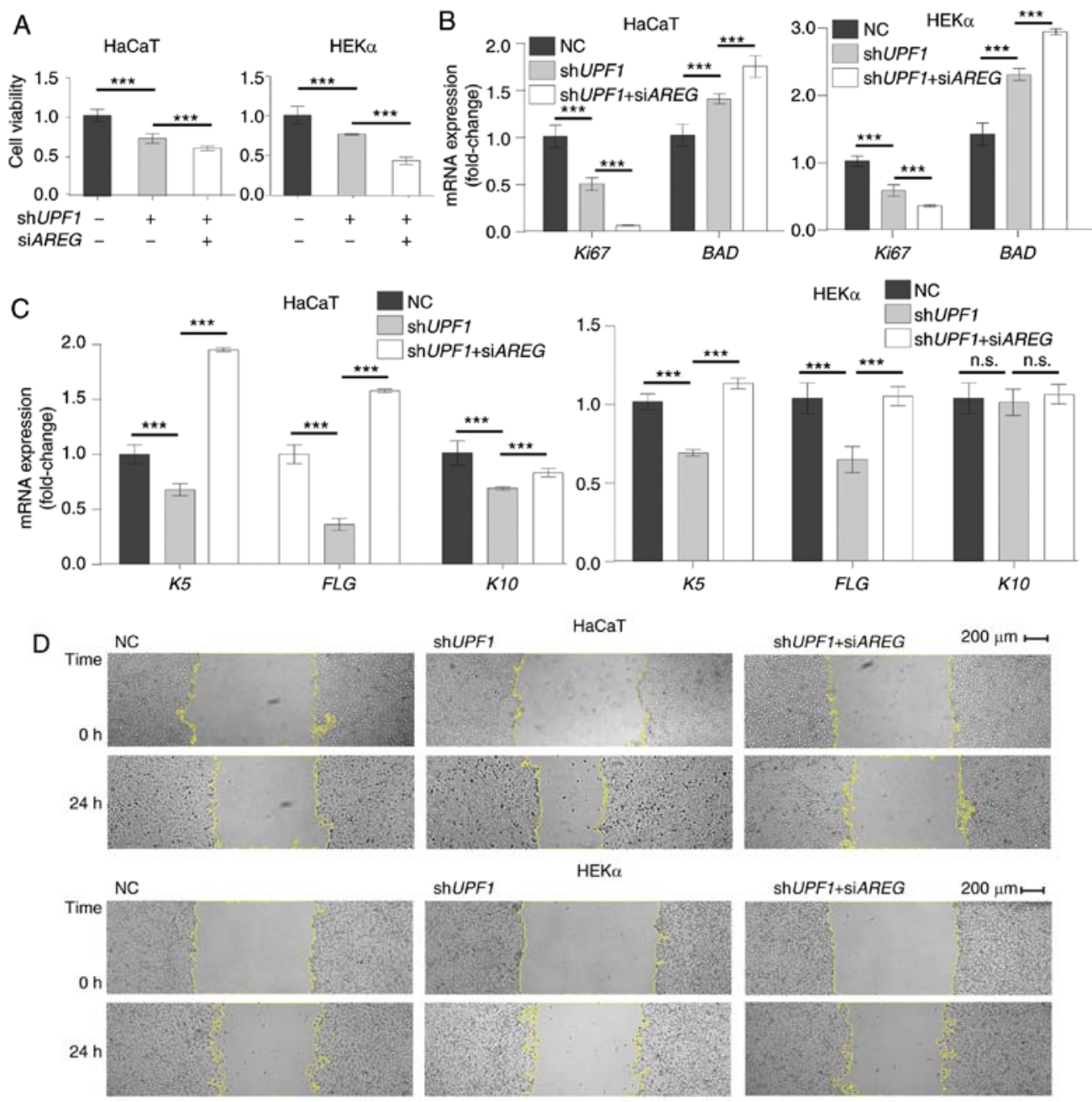

E
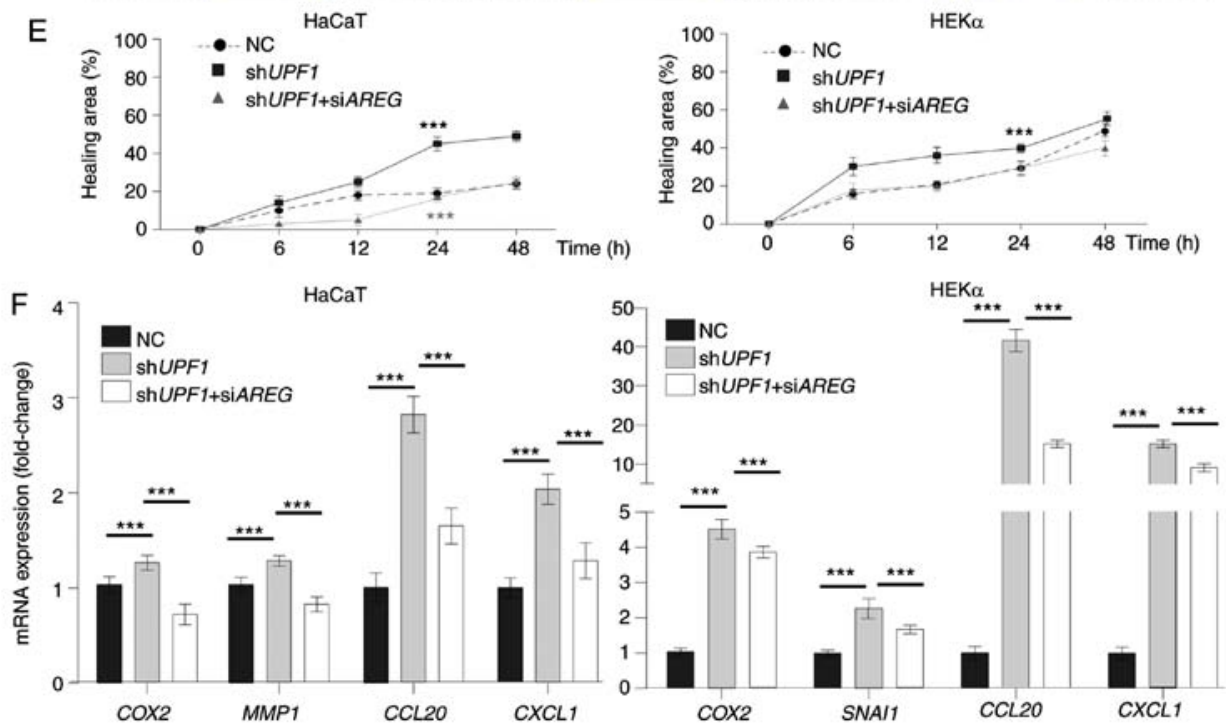

Figure 6. UPF1 depletion-induced AREG disturbs keratinocyte homeostasis. (A) Cell counting kit- 8 assays were performed to measure cell viability $48 \mathrm{~h}$ after UPF1 or AREG knockdown in HEK $\alpha$ and HaCaT cells. UPF1 and AREG depletion both decreased cell viability of keratinocytes. (B) The expression of Ki67 decreased in keratinocytes with $U P F 1$ or $A R E G$ knockdown, while the expression of apoptosis-related gene $B A D$ increased in both groups. (C) mRNA expression level of $K 5, F L G$ and $K 10$ was qualified by reverse transcription-quantitative PCR in keratinocytes. The expression levels of these differentiation makers decreased with UPF1 depletion and were rescued by supplementary AREG depletion. (D) Representative human keratinocyte scratch wounds with cells treated with the shUPF1 construct, or shUPF1 combined with siAREG. The wound images at 0 and $24 \mathrm{~h}$ are presented, and the epidermal sheet edge indicated by the yellow irregular curve was identified by ImageJ software automatically. UPF1 depletion induced the migration of keratinocytes by stabilization of $A R E G$. (E) Quantitative analysis of the measured wound coverage at $0,6,12,24$ and $48 \mathrm{~h} .{ }^{* * *} \mathrm{P}<0.001$ vs. NC group. (F) The motility markers $S N A I 1, C O X-2$ and $M M P 1$ and two chemokines associated with nuclear factor $\mathrm{\kappa B}$ activation, $C C L 20$ and $C X C L 1$, were identified at the mRNA level in keratinocyte cells. Expression levels of the migration makers and chemokines increased with $U P F 1$ depletion and were rescued by supplementary AREG depletion. The results are presented as the mean $\pm \mathrm{SD}$ from three independent experiments. ${ }^{* * *} \mathrm{P}<0.001$. UPF1, up-frameshift suppressor 1 homolog; $A R E G$, amphiregulin; BAD, BCL2 associated agonist of cell death; K5, keratin-5; FLG, filaggrin; K10, keratin-10; COX-2, cyclooxygenase-2; MMP1, matrix metalloproteinase 1; CCL20, chemokine (C-C motif) ligand 20; CXCL1, human recombinant GRO-alpha; NC, normal cells; Ki67, proliferation marker protein Ki67; n.s., not significant. 
By sanger sequencing in 5 scales, two somatic UPF1 transcripts were identified in 2 patients with sporadic psoriasis. The c.2935_2936insA and c.2030-2081del mutations in UPF1 mRNAs both disrupted the conventional reading frame and resulted in PTCs. The PTC-UPF1 transcripts should be immediately degraded by NMD under well-balanced RNA surveillance, while the abnormal mRNAs may escape degradation under exceptional circumstances, and consequently be translated into truncated proteins. Fully functional UPF1 is indispensable to the NMD pathway (1), while UPF1 without C-terminal domains has been assumed to fail in autophosphorylation or RNA unwinding (38). The truncated UPF1 protein disturbed NMD function, as demonstrated by the upregulation of the NMD-sensitive mRNAs NIK, TBL2 and NAT9. The calponin homology domain retained in truncated UPF1 protein has been shown to exert an inhibitory effect on helicase activity, and the inhibition can be relieved by UPF2 binding (39). Although in the current study RT-qPCR analysis showed that the expression of UPF1 WT was retained in the psoriasis scales, it was speculated that the truncated protein may competitively interact with UPF2 and disturb the helicase activity of residual full-length UPF1 protein. In $U P F 1$-depleted 293T cells, the mutated UPF1 exhibited slightly lower steady-state levels than UPFI WT, at both the mRNA and protein levels. One explanation for this result is that the mutants were destabilized by redundant endogenous UPF1. Of note, although the expression level of UPF1 was found to be increased in psoriasis data from the GEO database, IMQ-mice and psoriasis scale tissues, these data were derived from chip detection or RT-qPCR and depended on the specified nucleotide site binding, without considering the overall sequences of the transcripts or proteins. In fact, the increased expression of total UPF1 mRNA in psoriasis may be due to the accumulation of aberrant transcripts, at least in these 2 psoriasis scales. It remains to be elucidated whether the c.2935_2936ins A and c.2030_2081del UPF1 could be representative in psoriasis, and further data should be collected to verity this hypothesis. In the present study, the abnormal UPF1 transcripts, which were identified in two patients with sporadic psoriasis, were employed to reduce UPF1 activity. These finding indicated gene dysregulation related with NMD deficiency.

It was also concluded that $A R E G$ is an important substrate of the NMD pathway in keratinocytes. The EJ in the 3'UTR of $A R E G$ mRNA, which is present at low levels among cellular transcripts, could trigger the NMD pathway $(1,3)$. AREG has been reported to be the most abundant EGFR ligand in keratinocytes (30) and overexpressed in a wide spectrum of epithelial diseases, including squamous cell carcinoma of the head and neck, colon cancer, lung cancer and psoriasis (12-15). A negative association was identified between the expression levels of $A R E G$ and $U P F 1$ in keratinocytes, and the upregulation of $A R E G$ could be rescued by the recovery of $U P F I \mathrm{WT}$, rather than the mutated $U P F 1$. Based on the premise that post-transcriptional regulation of the $A R E G$ expression has little involvement in the NMD pathway, it was empirically determined in a half-life assay under inhibited UPF1 transcription in vitro that $A R E G$ mRNA was directly targeted by the NMD pathway. These results confirmed a previous hypothesis that $A R E G$ expression was regulated by NMD post-transcriptionally, rather than by impacting its transcription level (32).
In the current study, only AREG transcripts with the 3'UTR sequence could be regulated by NMD, and the results based on FLuc/RLuc dual-luciferase reporter system indicated that the 3'UTR sequence of AREG mRNA was sufficient to trigger the NMD pathway. It was therefore concluded that $A R E G$ mRNA was a primary NMD substrate, and that the mechanism through which AREG was degraded by NMD was dependent on its 3'UTR.

According to the classic NMD mechanism, termination codons situated $>50-55 \mathrm{nts}$ upstream of an EJ are typically expected to trigger mRNA surveillance, depending on a multi-subunit protein complex, the exon junction complex (EJC) (3). However, the distance between the termination codon (Ter) and the EJ downstream in the AREG is only $18 \mathrm{nts,}$ which may prevent the mRNA from assembling a stable EJC. According to a previous report, 50\% of EJCs were mapped to a non-canonical position, where no binding sites were predicted to exist (40), suggesting that the $A R E G$ might be targeted by NMD by a 3'UTR-dependent but EJC-independent pathway. The relative increase in the half-life of $A R E G$ mRNA in UPF1-depleted cells was just 1.23-fold, which was smaller than that of several documented substrates with a similar NMD-inducing feature (Table SV) (8). As the NMD pathway targets natural substrates in different magnitudes (8), it was speculated that the recognition reaction between $A R E G$ and the NMD pathway may be weaker compared with other endogenous substrates. AREG may contribute to diverse biological mechanisms depending on its dominance in keratinocytes, which is in line with the cell-specific character of NMD.

The NMD pathway governs different subsets of substrates and participates in diverse, essential homeostatic mechanisms in different cells (6), and the perturbation of NMD influences cellular homeostasis in a cell-specific manner. By contrast, aberrant UPF1 may lead to reprogramming towards a more malignant state in premalignant glandular pancreatic cells (9), and may drive a proinflammatory response in lung epithelial cells (7). As shown in HaCaT and HEKa keratinocytes used in the current study, UPF1 depletion decreased viability and differentiation, induced re-epithelialization, with increased expression of motility markers SNAII, COX-2 and $M M P 1$, and upregulated the expression of the chemokines CCL2O and CXCL1.

NMD deficiency has been reported to result in cell apoptosis though preferentially increasing the level of the growth arrest and DNA damage inducible b in 293 cells (33). Double Homeobox 4 mRNA, an NMD substrate, has also been shown to cause apoptosis in muscle cells (34). The BAD protein has been reported to be a proapoptotic factor inactivated by the AKT signaling (41). In the present study, the expression of $B A D$ mRNA was upregulated in the UPFI-depleted keratinocytes. As a major autocrine growth factor for human keratinocytes, AREG plays an important, positive role in keratinocyte proliferation (30). Unlike the previously reported role of $A R E G$ in inducing hyperproliferation by itself (11), the disruption of NMD, along with stabilization of AREG observed in the current study, decreased the proliferation of keratinocytes, and following the depletion of endogenous $A R E G$, the viability of keratinocytes further decreased, indicating an anti-apoptotic role of AREG. NMD has been shown to extensively impact gene expression either directly, by targeting degradation, or 
indirectly, via downstream cascades (1). NMD deficiency in keratinocytes may mediate a proapoptotic effect in advance and abrogate the proliferation-promoting effect of $A R E G$ alone, as shown in a previous study, in which oncogene-induced proliferation was inhibited by cellular senescence (42).

$A R E G$ is considered to prevent keratinocyte differentiation, and the suppression of $A R E G$ to irreversibly upregulate genes involved in keratinocyte differentiation $(11,43)$. The present data demonstrated that the depletion of UPF1 in HaCaT and HEK $\alpha$ cells downregulated the expression of differentiation markers by targeting AREG. Skin diseases, such as psoriasis, can be triggered by physical injury in susceptible patients (known as the Koebner phenomenon), and it is critical to understand the elements participating in processes occurring in cutaneous wounds $(44,45)$. Additionally, it has been suggested that endogenous $A R E G$ modulates tissue repair and regulates the migration of several cell types, such as breast cancer cells $(46,47)$. The current findings confirmed that NMD perturbation in keratinocytes promoted wound re-epithelialization by adjusting endogenous $A R E G$ levels. Previous reports have shown that the overexpression of exogenous $A R E G$ in keratinocytes does not result in marked increases in cell migration (48), and the contrasting results in the present study highlight the unique character of NMD deficiency in cellular balance. $A R E G$ has also been reported to mediate inflammatory action in keratinocytes $(49,50)$. In the present study, NMD normally suppressed inflammatory activation by targeting $A R E G$ mRNA; when this suppression was alleviated due to $U P F 1$ deficiency, the stabilization of AREG mRNA led to a high expression of the proinflammatory chemokines, which may contribute to triggering immune cell infiltration and angiogenesis.

The present data supported a model in which NMD normally regulated $A R E G$ abundance to maintain the homeostasis in keratinocytes. NMD deficiency caused by UPF1 alterations could disrupt proliferation, inhibit cell differentiation, promote wound healing, and activate inflammation response, by regulating $A R E G$ post-transcriptionally in keratinocytes, leading to indirect impacts on downstream gene cascades.

To the best of our knowledge, this is the first study revealing mutated UPF1 transcripts in psoriasis and investigating the relationship between the $A R E G$-NMD axis and homeostasis of keratinocytes. These findings of aberrant UPF1 expression in psoriasis and disruption of gene regulation at the post-transcriptional level might be useful for understanding the full development of keratinocyte morbidity. Furthermore, these results suggested that therapies involving NMD intervention may have the potential to ameliorate skin diseases or even other diseases related to abnormal cell differentiation, wound healing or inflammatory responses, through rescuing the dysregulation of multiple NMD substrates, such as AREG, either directly or via indirect regulation cascades. Mouse tissue samples were suitable for analysis; however, the human scale samples are fragile and were only selected due to the lack of availability of biopsy samples. The role of the $A R E G-\mathrm{NMD}$ axis in treated psoriasis is still unknown, as all patients recruited in the current study were not treated with any therapy for at least 2 weeks before the sampling, and it was challenging to follow the patients further without electronic medical records in the hospital system. In order to determine whether the mutated UPF1 could be representative in psoriasis, the quality and quantity of samples should be improved in the future, and an animal model with gene modification could be used for in vivo verification. In addition, the role of the $A R E G$-NMD axis in skin diseases with abnormal UPF1 expression levels, such as Marfan syndrome, non-melanoma skin cancer and malignant melanoma, should be investigated further.

\section{Acknowledgements}

The pCMV-MYC-UPF1 was provided by Professor Lynne E. Maquat (University of Rochester), the Phblv-U6-puro construct was provided by Professor Fan Handong (Hangzhou Normal University), and the pEGFP-N1 vector was provided by Dr Wang Miao (Hangzhou Normal University).

\section{Funding}

This research was funded by Natural Science Foundation of Zhejiang Province, China (grant no.LQ18H110001) and National Natural Science Foundation of China (grant no. 81802771).

\section{Availability of data and materials}

The datasets used or analyzed during the current study are available from the corresponding author on reasonable request.

\section{Authors' contributions}

YC and QL were contributors in acquisition of data. NS generated constructs for this study and contributed to revising the manuscript. QZ provided resources and performed data analysis. JR contributed to the construction of the mouse model and revised the manuscript. LL contributed analytical tools and was involved in the construction of the mouse model. LW contributed to designing the research and writing the original draft. CL designed the research, provided resources and was a major contributor in interpretation of data. All authors read and approved the final manuscript.

\section{Ethics approval and consent to participate}

Ethics approval for sampling from patients with psoriasis was obtained from the Ethics Committee of Hangzhou Normal University, Hangzhou, China (approval no. 2017-010). Tissues were scraped by blunt scalpels with patients' permission at the Affiliated Hospital of Ningbo University, Ningbo, China. Ethics approval for animal experimentation was obtained from the Ethics Committee of Hangzhou Normal University, Hangzhou, China (approval no. 2016008).

\section{Patient consent for publication}

Not applicable.

\section{Competing interests}

The authors declare that they have no competing interests. 


\section{References}

1. Karousis ED and Mühlemann O: Nonsense-mediated mRNA decay begins where translation ends. Cold Spring Harb Perspect Biol 11: a032862, 2019.

2. Huang L, Low A, Damle SS, Keenan MM, Kuntz S, Murray SF, Monia BP and Guo S: Antisense suppression of the nonsense mediated decay factor Upf $3 b$ as a potential treatment for diseases caused by nonsense mutations. Genome Biol 19: 4, 2018.

3. Jaffrey SR and Wilkinson MF: Nonsense-mediated RNA decay in the brain: Emerging modulator of neural development and disease. Nat Rev Neurosci 19: 715-728, 2018.

4. Celik A, He F and Jacobson A: NMD monitors translational fidelity 24/7. Curr Genet 63: 1007-1010, 2017.

5. Popp MW and Maquat LE: Nonsense-mediated mRNA decay and cancer. Curr Opin Genet Dev 48: 44-50, 2018.

6. Huang L, Lou CH, Chan W, Shum EY, Shao A, Stone E, Karam R, Song HW and Wilkinson MF: RNA homeostasis governed by cell type-specific and branched feedback loops acting on NMD. Mol Cell 43: 950-961, 2011.

7. Lu J, Plank TD, Su F, Shi X, Liu C, Ji Y, Li S, Huynh A, Shi C, Zhu B, et al: The nonsense-mediated RNA decay pathway is disrupted in inflammatory myofibroblastic tumors. J Clin Invest 126: 3058-3062, 2016.

8. Mendell JT, Sharifi NA, Meyers JL, Martinez-Murillo F and Dietz HC: Nonsense surveillance regulates expression of diverse classes of mammalian transcripts and mutes genomic noise. Nat Genet 36: 1073-1078, 2004.

9. Liu C, Karam R, Zhou Y, Su F, Ji Y, Li G, Xu G, Lu L, Wang C, Song M, et al: The UPF1 RNA surveillance gene is commonly mutated in pancreatic adenosquamous carcinoma. Nat Med 20: 596-598, 2014

10. Goetz AE and Wilkinson M: Erratum to: Stress and the nonsense-mediated RNA decay pathway. Cell Mol Life Sci 74: 4047, 2017.

11. Stoll SW, Johnson JL, Li Y, Rittié L and Elder JT: Amphiregulin carboxy-terminal domain is required for autocrine keratinocyte growth. J Invest Dermatol 130: 2031-2040, 2010.

12. Tinhofer I, Klinghammer K, Weichert W, Knödler M, Stenzinger A, Gauler T, Budach V and Keilholz U: Expression of amphiregulin and EGFRvIII affect outcome of patients with squamous cell carcinoma of the head and neck receiving cetuximab-docetaxel treatment. Clin Cancer Res 17: 5197-5204, 2011.

13. Shao J, Lee SB, Guo H, Evers BM and Sheng H: Prostaglandin E2 stimulates the growth of colon cancer cells via induction of amphiregulin. Cancer Res 63: 5218-5223, 2003.

14. Stabile LP, Rothstein ME, Keohavong P, Lenzner D, Land SR, Gaither-Davis AL, Kim KJ, Kaminski N and Siegfried JM Targeting of both the c-Met and EGFR pathways results in additive inhibition of lung tumorigenesis in transgenic mice. Cancers (Basel) 2: 2153-2170, 2010.

15. Cook PW, Pittelkow MR, Keeble WW, Graves-Deal R, Coffey RJ and Shipley GD: Amphiregulin messenger RNA is elevated in psoriatic epidermis and gastrointestinal carcinomas. Cancer Res 52: 3224-3227, 1992.

16. Cook PW, Piepkorn M, Clegg CH, Plowman GD, DeMay JM, Brown JR and Pittelkow MR: Transgenic expression of the human amphiregulin gene induces a psoriasis-like phenotype. J Clin Invest 9: 2286-2294, 2004.

17. Chung E, Cook PW, Parkos CA, Park YK, Pittelkow MR and Coffey RJ: Amphiregulin causes functional downregulation of adherens junctions in psoriasis. J Invest Dermatol 124: $1134-1140,2005$

18. Swindell WR, Xing X, Stuart PE, Chen CS, Aphale A, Nair RP, Voorhees JJ, Elder JT, Johnston A and Gudjonsson JE: Heterogeneity of inflammatory and cytokine networks in chronic plaque psoriasis. PLoS One 7: e34594, 2012.

19. Nair RP, Duffin KC, Helms C, Ding J, Stuart PE, Goldgar D, Gudjonsson JE, Li Y, Tejasvi T, Feng BJ, et al: Genome-wide scan reveals association of psoriasis with IL-23 and NF-kappaB pathways. Nat Genet 41: 199-204, 2009.

20. Reischl J, Schwenke S, Beekman JM, Mrowietz U, Stürzebecher S and Heubach JF: Increased expression of Wnt5a in psoriatic plaques. J Invest Dermatol 127: 163-169, 2007.

21. Yao Y, Richman L, Morehouse C, de los Reyes M, Higgs BW, Boutrin A, White B, Coyle A, Krueger J, Kiener PA and Jallal B: Type I interferon: Potential therapeutic target for psoriasis? PLoS One 3: e2737, 2008.
22. Suárez-Fariñas M, Li K, Fuentes-Duculan J, Hayden K, Brodmerkel C and Krueger JG: Expanding the psoriasis disease profile: Interrogation of the skin and serum of patients with moderate-to-severe psoriasis. J Invest Dermatol 132: 2552-2564, 2012.

23. Qiu CC, Su QS, Zhu SY and Liu RC: Identification of potential biomarkers and biological pathways in juvenile dermatomyositis based on miRNA-mRNA network. Biomed Res Int 2019: 7814287, 2019

24. Lv M, Deng J, Tang N, Zeng Y and Lu C: Efficacy and safety of tripterygium wilfordii Hook F on psoriasis vulgaris: A systematic review and meta-analysis of randomized controlled trials. Evid Based Complement Alternat Med 2018: 2623085, 2018.

25. Medicine DCoPLAAoTC: Consensus on diagnosis and treatment of Chinese integrative medicine for psoriasis vulgaris. Chin J Dermatol Venerol Integ Tradit West Med 8: 328, 2009.

26. Oka T, Sugaya M,Takahashi N, Takahashi T,Shibata S,MiyagakiT, Asano Y and Sato S: CXCL17 attenuates imiquimod-induced psoriasis-like skin inflammation by recruiting myeloid-derived suppressor cells and regulatory T cells. J Immunol 198: 3897-3908, 2017.

27. Livak KJ and Schmittgen TD: Analysis of relative gene expression data using real-time quantitative PCR and the 2(-Delta Delta C(T)) method. Methods 25: 402-408, 2001.

28. Ueyama A, Yamamoto M, Tsujii K, Furue Y, Imura C, Shichijo M and Yasui K: Mechanism of pathogenesis of imiquimod-induced skin inflammation in the mouse: A role for interferon-alpha in dendritic cell activation by imiquimod. J Dermatol 41: 135-143, 2014.

29. Amer M, Mostafa FF, Tosson Z and Nasr AN: Corneocytes in scaly parakeratotic diseases. Int J Dermatol 35: 417-421, 1996.

30. Stoll SW, Stuart PE, Lambert S, Gandarillas A, Rittié L, Johnston A and Elder JT: Membrane-tethered intracellular domain of amphiregulin promotes keratinocyte proliferation. J Invest Dermatol 136: 444-452, 2016.

31. Chan WK, Huang L, Gudikote JP, Chang YF, Imam JS, MacLean JA and Wilkinson MF: An alternative branch of the nonsense-mediated decay pathway. EMBO J 26: 1820-1830, 2007.

32. Lou CH, Shao A, Shum EY, Espinoza JL, Huang L, Karam R and Wilkinson MF: Posttranscriptional control of the stem cell and neurogenic programs by the nonsense-mediated RNA decay pathway. Cell Rep 6: 748-764, 2014.

33. Nelson JO, Moore KA, Chapin A, Hollien J and Metzstein MM: Degradation of Gadd45 mRNA by nonsense-mediated decay is essential for viability. Elife 5: e12876, 2016.

34. Feng Q, Snider L, Jagannathan S, Tawil R, van der Maarel SM, Tapscott SJ and Bradley RK: A feedback loop between nonsense-mediated decay and the retrogene DUX4 in facioscapulohumeral muscular dystrophy. Elife 4: 2015.

35. Li Y, Stoll SW, Sekhon S, Talsma C, Camhi MI, Jones JL, Lambert S, Marley H, Rittié L, Grachtchouk M, et al: Transgenic expression of human amphiregulin in mouse skin: Inflammatory epidermal hyperplasia and enlarged sebaceous glands. Exp Dermatol 25: 187-193, 2016.

36. Patel GK, Wilson CH, Harding KG, Finlay AY and Bowden PE: Numerous keratinocyte subtypes involved in wound re-epithelialization. J Invest Dermatol 126: 497-502, 2006.

37. Ruiz N, Wang B, Pentland A and Caparon M: Streptolysin O and adherence synergistically modulate proinflammatory responses of keratinocytes to group A streptococci. Mol Microbiol 27: 337-346, 1998.

38. Hurt JA, Robertson AD and Burge CB: Global analyses of UPF1 binding and function reveal expanded scope of nonsensemediated mRNA decay. Genome Res 23: 1636-1650, 2013.

39. Fiorini F, Boudvillain $M$ and Le HH: Tight intramolecular regulation of the human Upf1 helicase by its $\mathrm{N}$ - and C-terminal domains. Nucleic Acids Res 41: 2404-2415, 2013.

40. Schweingruber C, Rufener SC, Zünd D, Yamashita A and Mühlemann O: Nonsense-mediated mRNA decay-mechanisms of substrate mRNA recognition and degradation in mammalian cells. Biochim Biophys Acta 1829: 612-623, 2013.

41. Sun Y, Xiao S, Chen J, Wang M, Zheng Z, Song S and Zhang L: Heat shock protein 90 mediates the apoptosis and autophage in nicotinic-mycoepoxydiene-treated HeLa cells. Acta Biochim Biophys Sin (Shanghai) 47: 451-458, 2015.

42. Li X, Xu H, Xu C, Lin M, Song X, Yi F, Feng Y, Coughlan KA, Cho WC, Kim SS and Cao L: The yin-yang of DNA damage response: Roles in tumorigenesis and cellular senescence. Int J Mol Sci 14: 2431-2448, 2013. 
43. Stoll SW, Stuart PE, Swindell WR, Tsoi LC, Li B, Gandarillas A, Lambert S, Johnston A, Nair RP and Elder JT: The EGF receptor ligand amphiregulin controls cell division via FoxM1. Oncogene 35: 2075-2086, 2016.

44. Albanesi C, De Pità O and Girolomoni G: Resident skin cells in psoriasis: A special look at the pathogenetic functions of keratinocytes. Clin Dermatol 25: 581-588, 2007.

45. Lai Y, Li D, Li C, Muehleisen B, Radek KA, Park HJ, Jiang Z, Li Z, Lei H, Quan Y, et al: The antimicrobial protein REG3A regulates keratinocyte proliferation and differentiation after skin injury. Immunity 37: 74-84, 2012.

46. Zaiss DMW, Gause WC, Osborne LC and Artis D: Emerging functions of amphiregulin in orchestrating immunity, inflammation, and tissue repair. Immunity 42: 216-226, 2015.

47. Schmucker H, Blanding WM, Mook JM, Wade JF, Park JP, Kwist K, Shah $\mathrm{H}$ and Booth BW: Amphiregulin regulates proliferation and migration of HER2-positive breast cancer cells. Cell Oncol (Dordr) 41: 159-168, 2018.
48. Stoll SW, Rittié L, Johnson JL and Elder JT: Heparin-binding EGF-like growth factor promotes epithelial-mesenchymal transition in human keratinocytes. J Invest Dermatol 132: 2148-2157, 2012

49. Farley SM, Purdy DE, Ryabinina OP, Schneider P, Magun BE and Iordanov MS: Fas ligand-induced proinflammatory transcriptional responses in reconstructed human epidermis. Recruitment of the epidermal growth factor receptor and activation of MAP kinases. J Biol Chem 283: 919-928, 2008.

50. Kennedy-Crispin M, Billick E, Mitsui H, Gulati N, Fujita H, Gilleaudeau P, Sullivan-Whalen M, Johnson-Huang LM, Suárez-Fariñas M and Krueger JG: Human keratinocytes' response to injury upregulates CCL20 and other genes linking innate and adaptive immunity. J Invest Dermatol 132: 105-113, 2012.

This work is licensed under a Creative Commons Attribution-NonCommercial-NoDerivatives 4.0 International (CC BY-NC-ND 4.0) License. 